\title{
Average orders of multiplicative arithmetical functions of integer matrices
}

\author{
by \\ G. Bhowmik (Münster) and O. Ramaré (Princeton, N.J.)
}

1. Introduction. This paper is an attempt at being a part of the process of systematizing the study of the arithmetic of integer matrices. In this study a major departure from the case of multiplicative arithmetic on integers is the absence of commutativity which is both a cause of difficulty and interest.

One way of studying this arithmetic is to look at multiplicative arithmetical functions of integer matrices. In general, the pointwise evaluation of such functions is not simple, though certain functions have been completely (e.g. Euler $\phi$ function [3], [6]) or partially (e.g. the divisor functions $[6],[7],[2])$ evaluated. In special cases considered earlier, the analytic questions are often related to those raised in different contexts in arithmetic of integers (see, e.g. [4], [5]). Here we use the available knowledge on pointwise behaviour to study average orders of multiplicative functions, a problem for which we present some tools and ask some questions.

In Section 2 we present some necessary background material.

In Section 3 we determine the Dirichlet series associated with the convolution product of multiplicative functions on $2 \times 2$ matrices. The formula obtained (Theorem 1) is complicated. As a special case we are able to recover a previous result for the divisor function ([1], Theorem 5). We are also able to obtain average orders of other "natural" functions like the Euler $\phi$ function and the general divisor functions (see Corollaries 1-3).

We are unable to obtain an analogue of Theorem 1 for matrices of higher dimensions. Hence we concentrate on a more restricted problem which we expect to be generic, i.e. the average order of the divisor function. Here we have at our disposal a recurrence formula (Theorem 2). In Section 4 we use this to evaluate the divisor function pointwise for $3 \times 3$ matrices (Proposition 3 ) and the Dirichlet series associated with the function (Proposition 4).

Research of the first author partially supported by DAAD, Bonn.

Research of the second author partially supported by NSF grant DMS-9100383. 
In Section 5 we use the same recurrence formula to obtain the abscissa of convergence of the Dirichlet series associated with the divisor function of matrices of arbitrary size (Theorem 3).

Acknowledgements. The first author is grateful to Prof. V. C. Nanda at Chandigarh (India) and Prof. A. Krieg at Münster (FRG) for helpful discussions. The second author would like to acknowledge the hospitality of the Mathematisches Institut der Westfälischen Wilhelms-Universität, Münster, where this work was partially done.

2. Preliminaries. Most of the concepts mentioned here are on the lines of Nanda [7], [8]. Some of them have already appeared in print, but we include them nevertheless for the sake of readability. Latin upper case italics denote matrices and small Greek letters are used for functions.

Let $\mathbb{Z}(r, r)$ be the set of $r \times r$ non-singular integer matrices. For the purpose of arithmetic $\mathrm{GL}_{r}(\mathbb{Z})$, the group of units of $\mathbb{Z}(r, r)$, have to be handled carefully.

For $M, N \in \mathbb{Z}(r, r)$ to be considered equivalent (notation: $M \sim N$ ) there must exist $U, V \in \mathrm{GL}_{r}(\mathbb{Z})$ such that

$$
M=U N V .
$$

They are considered right equivalent if

$$
M=U N .
$$

Classical results due to H. J. S. Smith (1861) and C. Hermite (1851) assure us that every $M \in \mathbb{Z}(r, r)$ is

(a) equivalent to a unique diagonal matrix $S=\left(s_{i j}\right)$ in $\mathbb{Z}(r, r)$ where $s_{k k}=m_{1}(M) m_{2}(M) \ldots m_{k}(M)$, with $m_{i}(M) \in \mathbb{Z}^{+}$. We call $m_{i}(M)$ the $i$ th Smith invariant of $M$ and $S$ the Smith Normal Form (SNF) of $M$; and

(b) right equivalent to a unique lower triangular matrix $H=\left(h_{i j}\right)$ in $\mathbb{Z}(r, r)$ where $h_{k k} \in \mathbb{Z}^{+}$and $0 \leq h_{k+t, k}<h_{k k}$. We call $H$ the Hermite Normal Form (HNF) of $M$.

An arithmetical function $\chi$ is a mapping from $\mathcal{M}_{\mathbb{Z}}$, the set of integer matrices, to $\mathbb{C}$ such that

$$
\begin{gathered}
\chi(M)=\chi(N) \quad \text { if } M \sim N ; \quad \text { and } \\
\chi\left(\begin{array}{cc}
1 & 0 \\
0 & M
\end{array}\right)=\chi(M) .
\end{gathered}
$$

After preliminary considerations, it is enough to restrict the domain of $\chi$ to $\mathbb{Z}(r, r)$.

We note that arithmetical functions are completely determined pointwise if their values on SNF matrices are known. 
A (canonical) factorization of $M$ is defined as

$$
M=M_{1} M_{2}
$$

where $M_{2}$ is in HNF. We call $M_{2}$ a divisor of $M$ and use the notation $M_{2} \mid M$.

The divisor function $\tau^{(r)}(M)=\tau(M)$ counts the number of (inequivalent) factorizations of $M \in \mathbb{Z}(r, r)$, i.e.

$$
\tau^{(r)}(M)=\tau(M)=\sum_{M_{2} \mid M} 1
$$

It has been proved that $\tau$ is finite. We note that $\tau$ is a special member of a class of divisor functions $\sigma_{a}(M), a \in \mathbb{C}$, defined by

$$
\sigma_{a}^{(r)}(M)=\sigma_{a}(M)=\sum_{M_{2} \mid M}\left(\operatorname{det} M_{2}\right)^{a} .
$$

A multiplicative function $\chi$ is distributive over matrices with co-prime determinants, i.e.

$$
\chi(M N)=\chi(M) \chi(N) \quad \text { whenever }(\operatorname{det} M, \operatorname{det} N)=1 .
$$

We let $\Gamma$ be the set of all multiplicative arithmetical functions.

For pointwise evaluation of multiplicative functions it is enough to consider only diagonal matrices $F_{r}^{(p)}=F_{r}=\left(f_{i j}\right)$ in $\mathbb{Z}(r, r)$ where $p$ is a given prime number and $f_{k k}=p^{f_{1}+f_{2}+\ldots+f_{k}}$ for a positive integer $f_{1}$, and non-negative integers $f_{2}, \ldots, f_{k}$. We use the notation $\left\langle f_{1}, f_{2}, \ldots, f_{r}\right\rangle$ for $F_{r}$. Another matrix often used in pointwise evaluation of divisor functions is $\left\langle f_{1}, f_{2}, \ldots, f_{r}-1\right\rangle$ and is denoted by $G_{r}$.

We define the primes of $\mathbb{Z}(r, r)$ with respect to prime numbers $p$ and positive integers $j$ and $r, j \leq r$, as

$$
P_{j, r}^{(p)}=P_{r}=\left(\begin{array}{cc}
E_{r-j} & 0 \\
0 & p E_{j}
\end{array}\right)
$$

where $E$ and 0 denote identity and zero matrices respectively.

The Dirichlet convolution $*$ of two functions $\chi_{1}$ and $\chi_{2}$ is defined as

$$
\left(\chi_{1} * \chi_{2}\right)(M)=\sum_{M_{2} \mid M} \chi_{1}\left(M M_{2}^{-1}\right) \chi_{2}\left(M_{2}\right) .
$$

It has been proved that $(\Gamma, *)$ is an abelian group with its identity $\eta$ defined as

$$
\eta(M)= \begin{cases}1, & M \sim E, \\ 0, & \text { otherwise } .\end{cases}
$$

The unit function $\mathbf{1}$ is defined as

$$
\mathbf{1}(M)=1 \quad \forall M,
$$


so that

$$
\tau=\mathbf{1} * \mathbf{1}
$$

The Möbius function $\mu$ is defined as

$$
\mu * \mathbf{1}=\eta \text {. }
$$

For explicit evaluation, see [6].

We let $\nu(M)=\operatorname{det} M$, and define the Euler $\phi$ function as

$$
\phi=\mu * \nu .
$$

3. Dirichlet series. We can understand the convolution product by studying the associated Dirichlet series. We formally define $D_{H}\left(\chi ; s_{1}, s_{2}, \ldots\right.$ $\left.\ldots, s_{r}\right)$ as

$$
\begin{aligned}
D_{H}\left(\chi ; s_{1}, s_{2}, \ldots, s_{r}\right) & =\sum_{H \text { in HNF }} \frac{\chi(H)}{H^{s_{1}, \ldots, s_{r}}} \\
& =\sum_{H \text { in HNF }} \frac{\chi(H)}{m_{1}(H)^{s_{1}} \ldots m_{r}(H)^{s_{r}}} .
\end{aligned}
$$

The drawback of this definition is that, for $r \geq 2$, we do not have, in general, the property

$$
D_{H}\left(\chi_{1} * \chi_{2} ; s_{1}, \ldots, s_{r}\right)=D_{H}\left(\chi_{1} ; s_{1}, \ldots, s_{r}\right) D_{H}\left(\chi_{2} ; s_{1}, \ldots, s_{r}\right),
$$

which is a serious handicap. However, (3.2) holds if $s_{1}=r s_{r}, s_{2}=$ $(r-1) s_{r}, \ldots, s_{r-1}=2 s_{r}$, and this enabled us [2] to evaluate

$$
\sum_{\substack{H \text { in } H N F \\ \operatorname{det} H \leq x}} \chi(H)
$$

for some functions $\chi$.

However, since the value of $\chi$ depends only on SNF matrices, we can instead study

$$
D_{S}\left(\chi ; s_{1}, \ldots, s_{r}\right)=\sum_{S \text { in SNF }} \frac{\chi(S)}{S^{s_{1}, \ldots, s_{r}}}
$$

Clearly,

$$
D_{H}\left(\chi ; s_{1}, \ldots, s_{r}\right)=D_{S}\left(\chi h ; s_{1}, \ldots, s_{r}\right)
$$

where $h(S)$ is the number of HNF matrices equivalent to $S$. We now determine $D_{S}\left(\chi_{1} * \chi_{2} ; s_{1}, s_{2}\right)$.

3.1. Convolution product for $r=2$. Here we obtain the Dirichlet series $D_{S}\left(\chi_{1} * \chi_{2} ; s_{1}, s_{2}\right)$ for two multiplicative arithmetical functions $\chi_{1}$ and $\chi_{2}$. Since $\chi_{1} * \chi_{2}$ is multiplicative, it is enough to consider its value on $F_{2}$ (see 
Section 1). We first obtain all divisors of $F_{2}$ and introduce this in the " $p$ component" of $D_{S}\left(\chi_{1} * \chi_{2} ; s_{1}, s_{2}\right)$.

We require the following notations. The pair $\left(\langle k, s\rangle_{p},\langle l, r\rangle_{p}\right)$ is said to be a divisor couple of $F_{2}^{(p)}$ with multiplicity $m$ (the parameter $p$ is usually suppressed) if there exist $m$ distinct factorizations $A B$ of $F_{2}$ such that $\operatorname{SNF}(A)=\langle k, s\rangle$ and $\operatorname{SNF}(B)=\langle l, r\rangle$.

We now recall (see e.g. [9]) the SNF of a given matrix.

LEMMA 3.1.

$$
\operatorname{SNF}\left(\begin{array}{cc}
u & 0 \\
v & w
\end{array}\right)=\left(\begin{array}{cc}
\operatorname{gcd}(u, v, w) & 0 \\
0 & u w / \operatorname{gcd}(u, v, w)
\end{array}\right) .
$$

We now study the divisor couples of $F_{2}$. We introduce the notation

$$
\phi_{+}(n)=n \prod_{p \mid n} \frac{p+1}{p} .
$$

Proposition 1. Let $k, l, s, r$ be non-negative integers and $p$ a prime number. Then $\left(\langle k, s\rangle_{p},\langle l, r\rangle_{p}\right)$ is a divisor couple of $F_{2}^{(p)}$ if and only if $k+l \leq f_{1}$, and one of the following sets of conditions holds:

Case 1: $f_{2}=0 ; k+l+r=f_{1} ; s=r$. Here the multiplicity is $\phi_{+}\left(p^{r}\right)$.

Case 2: $f_{2} \neq 0$ and

(a) $k+l+r=f_{1} ; s=f_{2}+r$. Here the multiplicity is $p^{r}$.

(b) $k+l+r=f_{1}+t$ for $0<t<f_{2} ; s=f_{2}+r-2 t$. Here the multiplicity is $\phi\left(p^{r-t}\right)$.

(c) $k+l+r=f_{1}+f_{2} ; s=r-f_{2}$. Here the multiplicity is $p^{s}$.

Proof. Let us consider all factorizations of $F_{2}$, i.e.

$$
F_{2}=\left(\begin{array}{cc}
p^{f_{1}} & 0 \\
0 & p^{f_{1}+f_{2}}
\end{array}\right)=\left(\begin{array}{cc}
p^{f_{1}-a} & 0 \\
-x p^{f_{1}+f_{2}-b-a} & p^{f_{1}+f_{2}-b}
\end{array}\right)\left(\begin{array}{cc}
p^{a} & 0 \\
x & p^{b}
\end{array}\right)
$$

with $0 \leq x<p^{a}$ and $x p^{f_{1}+f_{2}-b-a}$ integral.

We use the notation $\langle\langle u, v\rangle\rangle$ for $\langle u, v-2 u\rangle$ and write $x$ as $x=p^{a-d} x^{\prime}$, with $\left(x^{\prime}, p\right)=1,0 \leq x^{\prime}<p^{d}, 0 \leq d \leq a, f_{1}+f_{2}-b-d \geq 0$ to get all divisor couples of $F_{2}$. (When $x=0$ we fix $d=0=x^{\prime}$.) We notice that all divisor couples are given by

$$
\begin{gathered}
\left(\left\langle\left\langle\min \left(f_{1}+f_{2}-b-d, f_{1}-a\right), 2 f_{1}+f_{2}-a-b\right\rangle\right\rangle,\langle\langle\min (b, a-d), a+b\rangle\rangle\right) \\
=\left(\left\langle\left\langle\min \left(f_{2}-b-d+a, 0\right)+f_{1}-a, 2 f_{1}+f_{2}-a-b\right\rangle\right\rangle,\right. \\
\left.\left\langle\left\langle\min \left(f_{2}-b-d+a, f_{2}\right)+b-f_{2}, a+b\right\rangle\right\rangle\right)
\end{gathered}
$$

with multiplicity $\phi\left(p^{d}\right)$. 
Now we look at the problem of determining the multiplicity of a divisor couple $(\langle k, s\rangle,\langle l, r\rangle)$ of $F_{2}$. We notice that for non-zero multiplicity

$$
k+l \leq f_{1}, \quad 2(k+l)+r+s=2 f_{1}+f_{2} .
$$

Now let $h=f_{2}-b-d+a$. Then we get

$$
k+l+r=\min (h, 0)-\min \left(h, f_{2}\right)+f_{1}+f_{2} .
$$

Since the graph of $h \rightarrow \min (h, 0)-\min \left(h, f_{2}\right)+f_{2}$ is

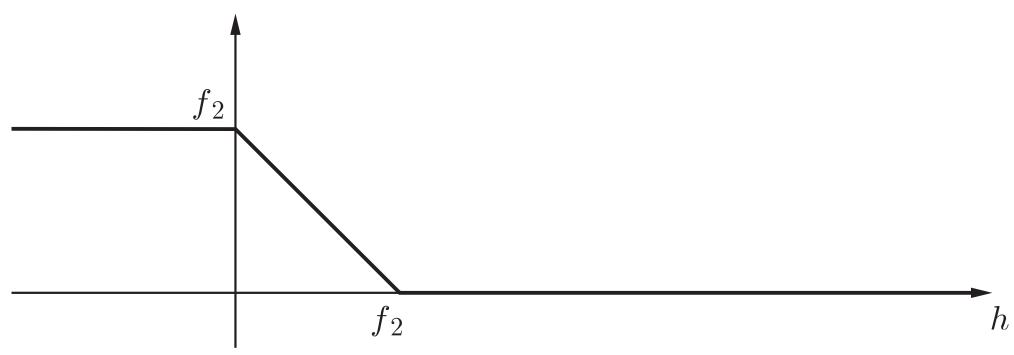

we consider the possibilities for $k+l+r-f_{1}$ corresponding to the various cases of our proposition.

Case 2: $f_{2} \neq 0$ and

(c) $k+l+r=f_{1}+f_{2}$. Then

$$
\begin{aligned}
k & =f_{1}+f_{2}-b-d, \\
s & =-f_{2}+b+2 d-a, \\
l & =a-d, \\
r & =b-a+2 d,
\end{aligned}
$$

with $d \geq f_{2}-b+a$; and the multiplicity is $\phi\left(p^{d}\right)$. This implies that $s=r-f_{2}$ and for all $d$ in $\left[0, f_{1}-(k+l)\right]$ there exist solutions.

(b) $k+l+r=f_{1}+t, 0<t<f_{2}$. Here $d=f_{1}-(k+l)$.

(a) $k+l+r=f_{1}$. This situation is similar to case $2(\mathrm{c})$.

Case 1: $f_{2}=0$. We take all solutions of cases $2(\mathrm{a})$ and $2(\mathrm{c})$ and subtract the solutions of case 2 (b) from it.

Remark. Proposition 1 gives us another method of evaluating $\tau\left(F_{2}\right)$.

We now reach the main result of this section.

THEOREM 1. Let $\chi_{1}$ and $\chi_{2}$ be two multiplicative arithmetical functions on $M_{2}(\mathbb{Z})$ and $p$ a prime number. Let 


$$
\begin{aligned}
& \Xi_{1}(r)=\sum_{k \geq 0} \chi_{1}\langle k, r\rangle_{p} X^{k}, \\
& \Xi_{2}(r)=\sum_{l \geq 0} \chi_{2}\langle l, r\rangle_{p} X^{l}
\end{aligned}
$$

for any non-negative integer $r$ and an indeterminate $X$. Then

$$
\begin{aligned}
\sum_{f_{1}, f_{2} \geq 0}( & \left.\chi_{1} * \chi_{2}\right) F_{2}^{(p)} X^{f_{1}} Y^{f_{2}} \\
= & \left(\sum_{r \geq 0} \Xi_{1}(r) Y^{r}\right)\left(\sum_{s \geq 0} \Xi_{2}(s) Y^{s}\right) \\
& +\left(1-\frac{Y^{2}}{p^{2} X}\right) \sum_{t \geq 1}\left(\frac{p X}{Y^{2}}\right)^{t}\left(\sum_{r \geq t} \Xi_{1}(r) Y^{r}\right)\left(\sum_{s \geq t} \Xi_{2}(s) Y^{s}\right) \\
& +\frac{1}{p}\left(\sum_{r \geq 1} \Xi_{1}(r) Y^{r}\right)\left(\sum_{s \geq 1} \Xi_{2}(s) Y^{s}\right) \\
& +\frac{1}{p} \sum_{t \geq 1}\left(\frac{p X}{Y^{2}}\right)^{t} \Xi_{1}(t) Y^{t} \Xi_{2}(t) Y^{t} .
\end{aligned}
$$

Proof. From Proposition 1 we get

$$
\begin{aligned}
& \sum_{f_{1}, f_{2} \geq 0}\left(\left(\chi_{1} * \chi_{2}\right) F_{2}\right) X^{f_{1}} Y^{f_{2}} \\
= & \sum_{k, r, l, s \geq 0} \chi_{1}\langle k, r\rangle \chi_{2}\langle l, s\rangle X^{k+l} Y^{r+s}\left\{\sum_{1}+\sum_{2}+\sum_{3}+\sum_{4}\right\}
\end{aligned}
$$

where

$$
\begin{aligned}
& \sum_{1}=\sum_{\substack{f_{1} \geq 0 \\
f_{2}=0}} X^{r} Y^{-2 r} \phi_{+}\left(p^{r}\right) \quad \text { if } r=s \text { and } 0 \text { if } r \neq s, \\
& \sum_{2}=\sum_{\substack{f_{1} \geq 0, f_{2}>0 \\
k+l+r=f_{1}+f_{2}, s=r-f_{2}}} p^{s} X^{s} Y^{-2 s}, \\
& \sum_{3}=\sum_{\substack{f_{1} \geq 0, f_{2}>0 \\
k+l+r=f_{1}, s=r+f_{2}}} p^{r} X^{r} Y^{-2 r}
\end{aligned}
$$

and

$$
\sum_{4}=\sum_{\substack{f_{1} \geq 0, f_{2}>0 \\ 0<m<f_{2}, k+l+r=f_{1}+m, s=f_{2}-2 m+r}} \phi\left(p^{r-m}\right) X^{r-m} Y^{2(m-r)} .
$$


We can thus write:

$$
\begin{aligned}
& \sum_{1}=\left\{\begin{array}{ll}
\sum_{0} X^{r} Y^{-2 r}\left\{2 p^{r}-\phi\left(p^{r}\right)\right\} & \text { if } r=s, \\
\sum_{2} & = \begin{cases}\sum_{0}\left(\frac{p X}{Y^{2}}\right)^{s} & \text { if } r>s,\end{cases} \\
\sum_{3} & \text { if } r \leq s, \\
\sum_{0}\left(\frac{p X}{Y^{2}}\right)^{r} & \text { if } r<s,
\end{array},\right. \\
& \text { if } r \geq s,
\end{aligned}
$$

and

$$
\sum_{4}= \begin{cases}\sum_{t=0}^{\min (r, s)-1} \frac{\phi\left(p^{t}\right)}{p^{t}}\left(\frac{p X}{Y^{2}}\right)^{t} & \text { if } \min (r, s)>0 \\ \text { otherwise. }\end{cases}
$$

We can verify that

$$
\sum_{t=0}^{y} \frac{\phi\left(p^{t}\right)}{p^{t}} T^{t}=\frac{1-T^{y+1}}{1-T}-\frac{T}{p(1-T)}\left(1-T^{y}\right) .
$$

Thus

$$
\begin{aligned}
& \sum_{f_{1}, f_{2} \geq 0}\left(\chi_{1} * \chi_{2}\right) F_{2} X^{f_{1}} Y^{f_{2}} \\
= & -\frac{1}{p} \sum_{\substack{k, l, r, s \geq 0 \\
\min (r, s)=0}} \chi_{1}\langle k, r\rangle X^{k} Y^{r} \chi_{2}\langle l, s\rangle X^{l} Y^{s} \\
+ & \frac{1}{p} \sum_{\substack{k, l \geq 0 \\
r>0}} \chi_{1}\langle k, r\rangle X^{k} Y^{r} \chi_{2}\langle l, r\rangle X^{l} Y^{r}\left(\frac{p X}{Y^{2}}\right)^{r} \\
+ & \frac{1-X / Y^{2}}{1-p X / Y^{2}}\left(\sum_{k, r \geq 0} \chi_{1}\langle k, r\rangle X^{k} Y^{r}\right)\left(\sum_{l, s \geq 0} \chi_{2}\langle l, s\rangle X^{l} Y^{s}\right) \\
- & \frac{p X / Y^{2}-1 / p}{1-p X / Y^{2}} \sum_{k, l, r, s \geq 0} \chi_{1}\langle k, r\rangle X^{k} Y^{r} \chi_{2}\langle l, s\rangle X^{l} Y^{s}\left(\frac{p X}{Y^{2}}\right)^{\min (r, s)} .
\end{aligned}
$$

Now, after introducing $\Xi_{1}(r), \Xi_{2}(s)$ and $t=\min (r, s)$ and going through some cumbersome manipulations we get the required formula.

We can use Theorem 1 to obtain Dirichlet series and average orders of various multiplicative functions. We give a few examples here: 
If $\chi_{1}=\mathbf{1}$, then

$$
\Xi_{1}(r)=\frac{1}{1-X}
$$

If $\chi_{1}=\mu$, then

$$
\Xi_{1}(r)= \begin{cases}1+p X & \text { if } r=0 \\ -1 & \text { if } r=1 \\ 0 & \text { if } r>1\end{cases}
$$

If $\chi_{1}=\nu^{a}$, then

$$
\Xi_{1}(r)=\frac{p^{a r}}{1-p^{2 a} X}
$$

From these we can verify that

$$
D_{S}\left(\eta ; s_{1}, s_{2}\right)=1
$$

and obtain some corollaries.

Corollary 1.

$$
D_{S}\left(\tau ; s_{1}, s_{2}\right)=\zeta\left(s_{1}\right)^{2} \zeta\left(s_{2}\right)^{2} \zeta\left(s_{1}-1\right) \prod_{p}\left(1+\frac{1}{p^{s_{1}}}-\frac{2}{p^{s_{1}+s_{2}}}\right) .
$$

Corollary 2.

$$
D_{S}\left(\phi ; s_{1}, s_{2}\right)=\zeta\left(s_{1}-2\right) \zeta\left(s_{2}-1\right) \prod_{p}\left(1-\frac{1}{p^{s_{1}}}-\frac{1}{p^{s_{2}}}+\frac{1}{p^{s_{1}+s_{2}+1}}\right)
$$

which further gives

$$
\sum_{\operatorname{det} S \leq x} \phi(S) \sim \frac{1}{2} \prod_{p}\left(1-\frac{1}{p^{2}}-\frac{1}{p^{4}}+\frac{1}{p^{5}}\right) x^{2} \log x .
$$

Corollary 3.

$$
D_{S}\left(\sigma_{a} ; s_{1}, s_{2}\right)
$$

$=\zeta\left(s_{1}\right) \zeta\left(s_{1}-2 a\right) \zeta\left(s_{2}\right) \zeta\left(s_{2}-a\right) \zeta\left(s_{1}-a-1\right) \prod_{p}\left(1+\frac{1}{p^{s_{1}-a}}-\frac{p^{a}+1}{p^{s_{1}+s_{2}-a}}\right)$.

4. The divisor function for $r=3$. For matrices of size greater than 2 , we concentrate only on the divisor function. We use a recurrence formula we have earlier obtained to explicitly evaluate $\tau\left(F_{3}\right)$ and then find a Dirichlet series corresponding to it. In more complicated cases, this recurrence does not seem to yield a closed formula. Its repeated use, however, helps us obtain the abscissa of convergence of the Dirichlet series for arbitrary $r$, which we do in the next section.

We recall that the matrix $G_{r}$ which occurs in the recurrence formula is a notation we use for a matrix of the form $\left\langle f_{1}, f_{2}, \ldots, f_{r}-1\right\rangle_{p}$. Our recurrence formula is: 
TheOrem $2[2]$.

$$
\sigma_{a}\left(F_{r}\right)-p^{a} \sigma_{a}\left(G_{r}\right)=\sigma_{a+1}\left(F_{r-1}\right) .
$$

We now use this theorem for $a=0, r=3$. But first we require the following proposition.

Proposition 2.

$$
\sigma_{1}\left(F_{2}\right)=\left(f_{1}+1\right) \frac{p^{2 f_{1}+f_{2}+1}}{p-1}-\frac{p^{2 f_{1}}}{p-1}-\frac{p^{2 f_{1}}-1}{(p-1)\left(p^{2}-1\right)} .
$$

Proof. We consider the factorization of $F_{2}$ :

$$
F_{2}=\left(\begin{array}{cc}
p^{f_{1}} & 0 \\
0 & p^{f_{1}+f_{2}}
\end{array}\right)=\left(\begin{array}{cc}
p^{f_{1}-t} & 0 \\
y & p^{f_{1}+f_{2}-u}
\end{array}\right)\left(\begin{array}{cc}
p^{t} & 0 \\
x & p^{u}
\end{array}\right)
$$

with $0 \leq x<p^{t}, y p^{t}+x p^{f_{1}+f_{2}-u}=0$.

The number of solutions of this system is $\left(p^{t}, p^{f_{1}+f_{2}-b}\right)$. We identify the following two cases:

Case 1: $t+u \leq f_{1}+f_{2}$. Here the contribution to $\sigma_{1}\left(F_{2}\right)$ comes from

$$
\sum_{t=0}^{f_{1}} \sum_{u=0}^{f_{1}+f_{2}-t} p^{2 t+u}
$$

Case 2: $t+u>f_{1}+f_{2}$. The contribution to $\sigma_{1}\left(F_{2}\right)$ is now made by

$$
\sum_{t=1}^{f_{1}} \sum_{u=f_{1}+f_{2}+1-t}^{f_{1}+f_{2}} p^{t+f_{1}+f_{2}}
$$

The sum of expressions (4.4) and (4.5) gives

$$
\sigma_{1}\left(F_{2}\right)=\left(f_{1}+1\right) p^{2 f_{1}} \sum_{n=0}^{f_{2}} p^{n}+\left(1+p^{-1}\right) \sum_{n=0}^{f_{1}}\left(f_{1}-n\right) p^{2 f_{1}-2 n-1} .
$$

But

$$
\sum\left(f_{1}-n\right) p^{2 f_{1}-2 n-1}=f_{1} p^{2 f_{1}-1} \sum p^{-2 n}-p^{2 f_{1}-1} \sum n p^{-2 n} .
$$

We now use the fact that

$$
\begin{aligned}
\sum_{m=1}^{t} m x^{m} & =x \sum_{m=1}^{t} m x^{m-1}=x \frac{d}{d x}\left\{\frac{1-x^{t+1}}{1-x}\right\} \\
& =x \frac{1-x^{t+1}}{(1-x)^{2}}-(t+1) \frac{x^{t+1}}{1-x}
\end{aligned}
$$

to get the result. 


\section{Proposition 3.}

$$
\begin{aligned}
\tau\left(F_{3}\right)= & \left.f_{1}+1\right)\left(f_{3}+1\right) \frac{p^{2 f_{1}+f_{2}+1}-p^{2 f_{1}}}{p-1} \\
& +2\left(f_{1}+1\right) \frac{p^{2 f_{1}+f_{2}+1}-p^{2 f_{1}}}{(p-1)^{2}}-2\left(f_{1}+1\right)\left(f_{2}+1\right) \frac{p^{2 f_{1}}}{p-1} \\
& +\left\{\left(\frac{1}{p}+1\right)\left(3 f_{1}+2 f_{2}+f_{3}+2\right)+\frac{2}{p}\right\} \\
& \times\left\{p \frac{1-p^{2 f_{1}+2}}{\left(p^{2}-1\right)^{2}}+\frac{\left(f_{1}+1\right) p^{2 f_{1}+1}}{p^{2}-1}\right\} \\
& -3(p+1)\left\{\frac{2 p^{2 f_{1}+4}}{\left(p^{2}-1\right)^{2}}-\left(2 f_{1}+3\right) \frac{p^{2 f_{1}+2}}{\left(p^{2}-1\right)^{2}}\right. \\
& \left.+\left(f_{1}+1\right)^{2} \frac{p^{2 f_{1}}}{p^{2}-1}-\frac{p^{2}+1}{\left(p^{2}-1\right)^{3}}\right\} .
\end{aligned}
$$
have

$$
\tau\left(F_{3}\right)=\tau\left\langle f_{1}, f_{2}-1,1\right\rangle+\left(f_{3}+1\right) \sigma_{1}\left(F_{2}\right) .
$$

But

$$
\tau\left\langle f_{1}, f_{2}-t, 1\right\rangle=\tau\left\langle f_{1}, f_{2}-t-1,1\right\rangle+2 \sigma_{1}\left\langle f_{1}, f_{2}-t\right\rangle
$$

for $0<t \leq f_{2}$.

Further, for $0<u<f_{1}$,

$$
\tau\left\langle f_{1}-u, 0,1\right\rangle=\tau\left\langle f_{1}-u-1,1,0\right\rangle+\sigma_{1}\left\langle f_{1}-u, 1\right\rangle+2 \sigma_{1}\left\langle f_{1}-u, 0\right\rangle .
$$

We use the above three substitutions to get

$$
\begin{aligned}
\tau\left(F_{3}\right)= & \left(f_{3}+1\right) \sigma_{1}\left(F_{2}\right) \\
& +2\left\{\sigma_{1}\left\langle f_{1}, f_{2}-1\right\rangle+\sigma_{1}\left\langle f_{1}, f_{2}-2\right\rangle+\ldots+\sigma_{1}\left\langle f_{1}, 0\right\rangle\right\} \\
& +\sigma_{1}\left\langle f_{1}-1,1\right\rangle+2 \sigma_{1}\left\langle f_{1}-1,0\right\rangle+\ldots+\sigma_{1}\langle 1,1\rangle \\
& +2 \sigma_{1}\langle 1,0\rangle+\tau\langle 1,0\rangle .
\end{aligned}
$$

Substituting the value of $\sigma_{1}$ from Proposition 2 now gives

$$
\begin{aligned}
\tau\left(F_{3}\right)= & \left(f_{1}+1\right) \sum_{m=0}^{f_{2}}\left(f_{3}+2 m+1\right) p^{2 f_{1}+f_{2}-m} \\
& +\sum_{n=0}^{f_{1}-1}\left\{( f _ { 1 } - n ) \left(\left(2 f_{2}+f_{3}+3 n+2\right) p^{2 f_{1}-2 n-1}\right.\right. \\
& \left.\left.+\left(2 f_{2}+f_{3}+3 n+4\right) p^{2 f_{1}-2 n-2}\right)\right\} .
\end{aligned}
$$


We write

$$
\begin{aligned}
\tau\left(F_{3}\right)= & \left(f_{1}+1\right)\left(f_{3}+1\right) p^{2 f_{1}} \sum_{t=0}^{f_{2}} p^{t}+2\left(f_{1}+1\right) p^{2 f_{1}+f_{2}} \sum_{t=0}^{f_{2}} t p^{-t} \\
& +\left\{\left(3 f_{1}+2 f_{2}+f_{3}+2\right)+p^{-1}\left(3 f_{1}+2 f_{2}+f_{3}+4\right)\right\} \sum_{t=0}^{f_{1}} t p^{2 t-1} \\
& -3\left(1+p^{-1}\right) \sum_{t=0}^{f_{1}} t^{2} p^{2 t-1}
\end{aligned}
$$

We now use (4.7) with $x=p^{-1}$ and $p^{2}$ to evaluate the second and third sums respectively. For the last sum we use the fact that

$$
\begin{aligned}
\sum_{m=1}^{t} m^{2} x^{m}= & x \frac{d^{2}}{d x^{2}} \sum_{m=1}^{t} x^{m+1}-\sum_{m=1}^{t} m x^{m} \\
= & 2 x \frac{1-x^{t+2}}{(1-x)^{3}}-2(t+2) \frac{x^{t+2}}{(1-x)^{2}} \\
& -\frac{(t+1)(t+2) x^{t+1}}{1-x}-\frac{x\left(1-x^{t+1}\right)}{(1-x)^{2}}+(t+1) \frac{x^{t+1}}{1-x}
\end{aligned}
$$

We now obtain the Dirichlet series corresponding to the divisor function for $r=3$. We use the notation:

$$
D\left(\tau^{(3)}\right)=\sum \tau\left(F_{3}^{(p)}\right) X^{f_{1}} Y^{f_{2}} Z^{f_{3}}
$$

for indeterminates $X, Y$ and $Z$, a prime number $p$ and non-negative integers $f_{1}, f_{2}, f_{3}$. We prove that

Proposition 4.

$$
\begin{aligned}
D\left(\tau^{(3)}\right)= & \frac{(1+Y+Z-Y Z)(1+p X-p(p+1) X Y)}{(1-X)\left(1-p^{2} X\right)^{2}(1-Y)(1-Z)} \\
& +\frac{(p+3) X+2 p X^{2}-\left(1+2(p+1) X+p X^{2}\right) Y}{1-X}
\end{aligned}
$$

Proof. We break $D\left(\tau^{(3)}\right)$ into several parts and use the recursion formula to make the computations possible, i.e.

$$
\begin{aligned}
D\left(\tau^{(3)}\right)= & \sum_{\substack{f_{1}, f_{2} \geq 0 \\
f_{3}>0}} \tau\left\langle f_{1}, f_{2}, f_{3}\right\rangle X^{f_{1}} Y^{f_{2}} Z^{f_{3}} \\
& +\sum_{\substack{f_{1} \geq 0 \\
f_{2}>0}} \tau\left\langle f_{1}, f_{2}, 0\right\rangle X^{f_{1}} Y^{f_{2}}+\sum_{f_{1} \geq 0} \tau\left\langle f_{1}, 0,0\right\rangle X^{f_{1}} .
\end{aligned}
$$

We denote the three sums on the right hand side of (4.18) by $H^{(3)}, H_{0}^{(2)}$ and 
$H_{00}^{(1)}$ respectively. Now,

$$
\begin{aligned}
H^{(3)}= & \sum \tau\left\langle f_{1}, f_{2}, f_{3}-1\right\rangle X^{f_{1}} Y^{f_{2}} Z^{f_{3}} \\
& +\frac{Z}{1-Z} \sum \sigma_{1}\left\langle f_{1}, f_{2}\right\rangle X^{f_{1}} Y^{f_{2}} \\
= & Z H^{(3)}+Z H_{0}^{(2)}+Z H_{00}^{(1)}+\frac{Z}{1-Z} K^{(2)}
\end{aligned}
$$

where $K^{(2)}=\sum_{f_{1}, f_{2} \geq 0} \sigma_{1}\left\langle f_{1}, f_{2}\right\rangle X^{f_{1}} Y^{f_{2}}$. Thus

$$
H^{(3)}=\frac{Z}{1-Z}\left(H_{0}^{(2)}+H_{00}^{(1)}\right)+\frac{Z}{(1-Z)^{2}} K^{(2)} .
$$

But $H_{0}^{(2)}=\sum \tau\left\langle f_{1}, f_{2}-1,1\right\rangle X^{f_{1}} Y^{f_{2}}+K^{(2)}-K_{0}^{(1)}$.

Writing

$$
H_{1}^{(2)}=\sum_{f_{1}, f_{2} \geq 0} \tau\left\langle f_{1}, f_{2}, 1\right\rangle X^{f_{1}} Y^{f_{2}}
$$

and

we get

$$
K_{0}^{(1)}=\sum_{f_{1} \geq 0} \sigma_{1}\left\langle f_{1}, 0\right\rangle X^{f_{1}}
$$

$$
H_{0}^{(2)}=H_{1}^{(2)} Y+K^{(2)}-K_{0}^{(1)}
$$

and

$$
H_{1}^{(2)}=H_{0}^{(2)}+K^{(2)}-K_{0}^{(1)} .
$$

Simplifying, we get

$$
H_{0}^{(2)}=\frac{1+Y}{1-Y} K^{(2)}-\frac{1}{1-Y} K_{0}^{(1)} .
$$

Using (4.20) and (4.23) we can write

$$
\begin{aligned}
D\left(\tau^{(3)}\right)=\left(\frac{1+Y}{1-Y} \cdot \frac{1}{1-Z}\right. & \left.+\frac{Z}{1-Z}\right) K^{(2)} \\
& -\frac{1}{1-Z} \cdot \frac{1}{1-Y} K_{0}^{(1)}+\frac{1}{1-Z} H_{0}^{(1)} .
\end{aligned}
$$

With similar manipulations, we can express $H_{00}^{(1)}$ in terms of the $K$ 's. In fact, with $K_{1}^{(1)}=\sum_{f_{1} \geq 0} \sigma_{1}\left\langle f_{1}, 1\right\rangle X^{f_{1}}$, we get

$$
H_{00}^{(1)}=\frac{1+X}{1-X} K_{0}^{(1)}+\frac{X}{1-X} K_{1}^{(1)}
$$

so that $D\left(\tau^{(3)}\right)$ can now be written in terms of the $K$ 's.

To evaluate the $K$ 's we use the recurrence further and express them in terms of $L^{(1)}=\sum \sigma_{2}\left\langle f_{1}\right\rangle X^{f_{1}}$. 
Thus

$$
K_{0}^{(1)}=p X K_{1}^{(1)}+L^{(1)}
$$

and

$$
K_{1}^{(1)}=p K_{0}^{(1)}+L^{(1)},
$$

which gives

$$
K_{0}^{(1)}=\frac{1+p X}{1-p^{2} X} L^{(1)}, \quad K_{1}^{(1)}=\frac{1+p}{1-p^{2} X} L^{(1)} .
$$

Now

$$
\begin{aligned}
K^{(2)} & =p \sum \sigma\left\langle f_{1}, f_{2}-1\right\rangle X^{f_{1}} Y^{f_{2}}+\frac{Y}{1-Y}+L^{(1)}+K_{0}^{(1)} \\
& =\frac{1+p X-p(p+1) X Y}{(1-p Y)(1-Y)\left(1-p^{2} X\right)} L^{(1)}
\end{aligned}
$$

But

$$
L^{(1)}=\sum\left(\frac{p^{2 f_{1}+2}-1}{p^{2}-1}\right) X^{f_{1}}=\frac{1}{1-X} \cdot \frac{1}{1-p^{2} X} .
$$

We have now evaluated all the sums.

Remark. From Proposition 4 we can derive that $\sum_{|s| \leq x} \tau^{(3)}(S) \sim$ $A x \log ^{4} x$ for a positive constant $A$.

5. A problem in higher rank. We are not able to find the Dirichlet series associated with the convolution product of matrices for $r \geq 3$. Hence we study the divisor function and with the help of the recursion formula (Theorem 2) obtain the abscissa of convergence of the associated Dirichlet series.

We define $\beta_{r}$ to be the abscissa of convergence of the Dirichlet series with positive coefficients:

$$
D_{S}^{0}(\tau, s)=\sum_{S \text { in SNF }} \frac{\tau(S)}{(\operatorname{det} S)^{s}}=D_{S}(\tau ; r s,(r-1) s, \ldots, s) .
$$

It is simpler to see the relative strength of our results for $D_{S}^{0}(\tau, s)$ though the methods employed help us understand $D_{S}\left(\tau ; s_{1}, \ldots, s_{r}\right)$ as well. Knowing the estimates $([1],[2])$

$$
\begin{aligned}
& \sum_{\substack{\operatorname{det} H \leq x \\
H \text { in HNF }}} \tau(H) \sim C_{r} x^{r} \log x ; \quad \sum_{\substack{\operatorname{det} H \leq x \\
H \text { in HNF }}} 1 \sim C_{r}^{\prime} x^{r} ; \\
& \sum_{\substack{\operatorname{det} S \leq x \\
S \text { in SNF }}} 1 \sim C_{r}^{\prime \prime} x ; \quad C_{r}, C_{r}^{\prime}, C_{r}^{\prime \prime} \text { positive constants }
\end{aligned}
$$


one would expect $\sum_{\operatorname{det} S \leq x, S \text { in SNF}} \tau(S)$ to be of order $x \log x$. But this argument is misleading even for $r=2$ and further so as $r \rightarrow \infty$ (see Theorem 3 ). We get an upper bound for $\tau$ in Proposition 5, the proof of which depends on a repeated use of our recurrence formula, which we do in the following lemmas. Henceforth we assume $a$ to be non-negative.

LEMMA 5.1.

$$
\begin{aligned}
& \sigma_{a}^{(r)}\left\langle f_{1}, \ldots, f_{r}\right\rangle \\
& \quad \leq p^{a f_{r}} \sigma_{a}^{(r)}\left\langle f_{1}, \ldots, f_{r-1}, 0\right\rangle+f_{r} p^{a\left(f_{r}-1\right)} \sigma_{a+1}^{(r-1)}\left\langle f_{1}, \ldots, f_{r-1}\right\rangle .
\end{aligned}
$$

Proof. We use the recurrence formula (Theorem 2) and the fact that $\sigma_{a}$ is non-negative.

When necessary, we use the notation $0_{m}$ to denote a string of $m$ zeros.

LEMMA 5.2. For $t \geq 1$,

$$
\begin{aligned}
p^{a t} \sigma_{a}^{(r)}\left\langle f_{1}, f_{2}, \ldots, f_{s-1}, f_{s}-1,0_{t-1},\right. & 1,0, \ldots, 0\rangle \\
& \leq \sigma_{a}^{(r)}\left\langle f_{1}, \ldots, f_{s}, 0, \ldots, 0\right\rangle .
\end{aligned}
$$

Proof. We have $p^{a} \sigma_{a}^{(r)}\left\langle f_{1}, \ldots, f_{s-1}, f_{s}-1,1,0, \ldots, 0\right\rangle \leq \sigma_{a}^{(r)}\left\langle f_{1}, \ldots, f_{s}\right.$, $0, \ldots, 0\rangle$. We now use induction on $t$.

LEMMA 5.3.

$$
\begin{aligned}
\sigma_{a}^{(r)}\left\langle f_{1}, \ldots, f_{s}, 0, \ldots, 0\right\rangle \leq & p^{a(r-s+1)} \sigma_{a}^{(r)}\left\langle f_{1}, \ldots, f_{s-1}, f_{s}-1,0, \ldots, 0\right\rangle \\
& +(r-s+1) \sigma_{a+1}^{(r-1)}\left\langle f_{1}, \ldots, f_{s}, 0, \ldots, 0\right\rangle
\end{aligned}
$$

Proof. A repeated use of the recurrence formula gives

$$
\begin{aligned}
& \sigma_{a}^{(r)}\left\langle f_{1}, \ldots, f_{s}, 0, \ldots, 0\right\rangle \\
= & p^{a(r-s+1)} \sigma_{a}^{(r)}\left\langle f_{1}, \ldots, f_{s-1}, f_{s}-1,0, \ldots, 0\right\rangle \\
& +\sigma_{a+1}^{(r-1)}\left\langle f_{1}, \ldots, f_{s}, 0, \ldots, 0\right\rangle+p^{a} \sigma_{a+1}^{(r-1)}\left\langle f_{1}, \ldots, f_{s}-1,1,0, \ldots, 0\right\rangle \\
& +\ldots+p^{a(r-s)} \sigma_{a+1}^{(r-1)}\left\langle f_{1}, \ldots, f_{s}-1,0, \ldots, 0\right\rangle .
\end{aligned}
$$

We now use Lemma 5.2.

Lemma 5.4. For $1 \leq s \leq r-1$,

$$
\begin{aligned}
& \sigma_{a}^{(r)}\left\langle f_{1}, \ldots, f_{s}, 0, \ldots, 0\right\rangle \\
& \leq p^{a(r-s+1) f_{s}} \sigma_{a}^{(r)}\left\langle f_{1}, \ldots, f_{s-1}, 0, \ldots, 0\right\rangle \\
& \quad+(r-s+1) f_{s} \max \left(1, p^{(a-r+s)\left(f_{s}-1\right)}\right) \sigma_{a+1}^{(r-1)}\left\langle f_{1}, \ldots, f_{s}, 0, \ldots, 0\right\rangle .
\end{aligned}
$$

Proof. We get this by a repeated use of the previous lemma. 
LEMMA 5.5.

$$
\begin{aligned}
\leq & p^{a\left(f_{r}+2 f_{r-1}+\ldots+r f_{1}\right)} \\
& +\left(f_{r}+2 f_{r-1}+\ldots+r f_{1}\right) \max _{1 \leq s \leq r}\left(p^{\Sigma_{t=s}^{r}(a-r+t) f_{t}}\right) \sigma_{a+1}^{(r-1)}\left\langle f_{1}, \ldots, f_{r-1}\right\rangle .
\end{aligned}
$$

Pr o of. From Lemma 5.1 we get

$$
\begin{aligned}
\sigma_{a}^{(r)}\left\langle f_{1}, \ldots, f_{r}\right\rangle \\
\quad \leq p^{a f_{r}} \sigma_{a}^{(r)}\left\langle f_{1}, \ldots, f_{r-1}, 0\right\rangle+f_{r} p^{a f_{r}} \sigma_{a+1}^{(r-1)}\left\langle f_{1}, \ldots, f_{r-1}\right\rangle .
\end{aligned}
$$

We then use Lemma 5.4 repeatedly to get the upper bound

$$
\begin{aligned}
p^{a\left(f_{r}+2 f_{r-1}+\ldots+r f_{1}\right)} & +\sum_{s=1}^{r}(r-s+1) f_{s} \max \left(1, p^{(a-r+s)\left(f_{s}-1\right)}\right) \\
& \times p^{a\left(f_{r}+2 f_{r-1}+\ldots+(r-s) f_{s+1}\right)} \sigma_{a+1}^{(r-1)}\left\langle f_{1}, \ldots, f_{s}, 0, \ldots, 0\right),
\end{aligned}
$$

where for $s=r, \sigma_{a+1}^{(r-1)}\left\langle f_{1}, \ldots, f_{s}, 0, \ldots, 0\right\rangle$ is to be interpreted as $\sigma_{a+1}^{(r-1)}\left\langle f_{1}, \ldots, f_{r-1}\right\rangle$. Now a repeated use of Lemma 5.2 gives

$$
\begin{aligned}
\sigma_{a+1}^{(r-1)}\left\langle f_{1}\right. & \left., \ldots, f_{s}, 0, \ldots, 0\right\rangle \\
& \leq p^{(a+1)\left(f_{r-1}+2 f_{r-2}+\ldots+(r-s-1) f_{s+1}\right)} \sigma_{a+1}\left\langle f_{1}, \ldots, f_{r-1}\right\rangle .
\end{aligned}
$$

Hence the lemma.

We are now ready for our proposition. We use the notations $\lceil x\rceil$ for the integer $n$ satisfying

$$
n-1<x \leq n,
$$

and $\alpha_{r}(t)$ to denote

$$
\left\lceil\frac{r+1-t}{2}\right\rceil\left(r+1-t-\left\lceil\frac{r+1-t}{2}\right\rceil\right) .
$$

Proposition 5.

$$
\tau\left\langle f_{1}, \ldots, f_{r}\right\rangle \leq 1+\left(f_{r}+2 f_{r-1}+\ldots+r f_{1}\right)^{r-1} p^{\Sigma_{t=1}^{r} \alpha_{r}(t) f_{t}} .
$$

Proof. We use Lemma 5.5 repeatedly, beginning with $a=0$. The maximum involved is easily computable, and we obtain a sum of various $p^{m}$ 's. These exponents $m$, for some $0 \leq k<r$, are

$$
\sum_{t=1}^{r} \sum_{\substack{a=(r+1-t) / 2 \\ a \leq k}}^{r-t}(t-r+2 a) f_{t}+ \begin{cases}(k+1)(r-k-t) f_{t} & \text { if } t<r-k \\ 0 & \text { otherwise }\end{cases}
$$


If $t \geq r-k$, the coefficient of $f_{t}$ is given by

$$
\left\lceil\frac{r+1-t}{2}\right\rceil\left(r+1-t-\left\lceil\frac{r+1-t}{2}\right\rceil\right)=\alpha_{r}(t) .
$$

If $t<r-k$, the coefficient is also

$$
\begin{aligned}
\left(k+1-\left\lceil\frac{r+1-t}{2}\right\rceil\right)\left(k+t-r+\left\lceil\frac{r+1-t}{2}\right\rceil\right) \\
+(k+1)(r-k-t)=\alpha_{r}(t) .
\end{aligned}
$$

The above proposition, together with a previous result [6] for $\tau\left(P_{r}\right)$, i.e.

$$
\tau^{(r)}\langle 1,0, \ldots, 0\rangle=\sum_{j=0}^{r}\left[\begin{array}{l}
r \\
j
\end{array}\right],
$$

where the $\left[\begin{array}{l}r \\ j\end{array}\right]$ are Gaussian polynomials in $p$, help us get the abscissa of convergence of $D_{S}^{0}(\tau, s)$.

TheOREM 3. For a positive integer $r$,

$$
\beta_{r}=\frac{\alpha_{r}(1)+1}{r} .
$$

P r o of. The upper bound for $\beta_{r}$ comes from Proposition 5, and the lower bound from (5.11).

A closer look at the foregoing proofs in fact shows that

$$
x^{\beta_{r}} \ll \sum_{\substack{\operatorname{det} S \leq x \\ S \text { in SNF }}} \tau(S) \ll x^{\beta_{r}} \log ^{\gamma_{r}} x,
$$

for some $\gamma_{r} \geq 0$.

\section{References}

[1] G. Bhow mik, Average orders of certain functions connected with arithmetic of matrices, J. Indian Math. Soc. 60 (1993), to appear.

[2] - Divisor functions of integer matrices: evaluations, average orders and applications, Astérisque 209 (1992), 169-177.

[3] U. Christian, Über teilerfremde symmetrische Matrizenpaare, J. Reine Angew. Math. 229 (1968), 43-49.

[4] J. Knopfmacher, Solomon's zeta function and enumeration of lattices over orders, Analysis 5 (1985), 29-42.

[5] E. Krätzel, Estimates in the general divisor problem, Abh. Math. Sem. Univ. Hamburg 62 (1992), 191-206.

[6] V. C. Nand a, Arithmetic functions of matrices and polynomial identities, in: Colloq. Math. Soc. János Bolyai 34, North-Holland, 1984, 1107-1126.

[7] -, On arithmetical functions of integer matrices, J. Indian Math. Soc. 55 (1990), $175-188$. 
[8] V. C. Nanda, Arithmetic of matrices, manuscript.

[9] M. New man, Integer Matrices, Academic Press, New York, 1972.

MATHEMATISCHES INSTITUT WESTFÄLISCHE WILHELMS-UNIVERSITÄT EINSTEINSTRASSE 62 48149 MÜNSTER, B.R.D
SCHOOL OF MATHEMATICS INSTITUTE FOR ADVANCED STUDY PRINCETON, NEW JERSEY 08540 U.S.A. 